\title{
Effect of intraoperative use of dexmedetomidine on post-operative analgesia in patients undergoing elective spine surgery under general anaesthesia
}

\author{
Madhumita Ray ${ }^{1}$, Chanchal Kumar Dalai' ${ }^{2}$, Shah Newaz Ahmed ${ }^{3}$, Amita Acharjee ${ }^{4}$ \\ ${ }^{1}$ Associate Professor, Department Of Anaesthesiology, College Of Medicine and JNM Hospital, WBUHS, ,Kalyani, \\ Nadia, West Bengal, India, ${ }^{2}$ Associate Professor, ${ }^{3}$ Demonstrator, Department Of Pharmacology, College Of Medicine \\ and JNM Hospital, WBUHS, ,Kalyani, Nadia, West Bengal, India, ${ }^{4}$ Professor and Head Of The Department, \\ Department Of Neuro-anaesthesiology, Bangur Institute of Neurosciences, Kolkata, West Bengal, India
}

Background: Dexmedetomidine is an alpha2 agonist which shows sedative, analgesic and anti-adrenergic effects and is useful as an adjuvant agent in general anaesthesia (GA) in major surgeries. Aims and Objectives: In this study, we evaluated the analgesic efficacy of intra-operative infusion of dexmedetomidine in alleviating post-operative pain in elective spine surgery using multiple efficacy parameters. Materials and Methods: Patients $(n=60)$ scheduled to undergo elective spine surgery under GA were randomised to receive either dexmedetomidine (treatment arm) or normal saline (control arm) in the intra-operative period. The analgesic efficacy of the drug in the post-operative period was assessed using- the time to first rescue analgesic, total requirement of analgesic, Visual Analog Scale (VAS) score and the number of back-up analgesic required. Results: There was no statistically significant difference in the baseline clinical and demographic characteristics between the two groups. The time to first rescue analgesic, total requirement of analgesic, Visual Analog Scale (VAS) score and the number of back-up analgesic required, were all statistically significant in favour of the treatment arm $(P<0.001)$. Conclusion: Dexmedetomidine provides effective postoperative analgesia and reduces tramadol requirements in elective spine surgery performed under general anaesthesia.

Key words: Dexmedetomidine; General anaesthesia; Analgesia; Spine surgery

\section{Access this article online}

Website:

http://nepjol.info/index.php/AJMS DOI: 10.3126/ajms.v12i5.34151

E-ISSN: 2091-0576

P-ISSN: 2467-9100

Copyright (c) 2021 Asian Journal of Medical Sciences

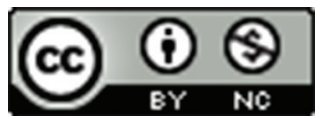

This work is licensed under a Creative Commons Attribution-NonCommercial 4.0 International License.

\section{INTRODUCTION}

Dexmedetomidine, an alpha-2 agonist, initially approved as a short acting analgesic and hypnotic, is being successfully used as an adjuvant agent in general anaesthesia. The drug reduces the requirement of the primary anaesthetic drugs during general anaesthesia in major surgeries, and also reduces analgesic requirement in the post-operative period. ${ }^{1}$ In spine surgeries, which are generally prolonged and accompanies painful episodes in the post-operative period, dexmedetomidine have been shown to reduce both the intra-operative anaesthetic and post-operative analgesic requirement. ${ }^{2}$ However, studies on the post-operative analgesic effect of dexmedetomidine are few, and further studies are required to establish the unequivocal role of the drug in attenuating post-operative pain in spine surgery. In our study, we assessed the role of intra-operative infusion of dexmedetomidine in alleviating post-operative pain in elective spine surgery within 24 hours of surgery using multiple efficacy parameters.

\section{MATERIALS AND METHODS}

The study was a randomised, placebo controlled, addon trial, conducted to assess the efficacy and safety 
of intraoperative use of dexmedetomidine in patients undergoing elective spine surgery. The primary analysis consisted of the effect of dexmedetomidine in reducing the dose of anaesthetic medications during general anaesthesia. The secondary analysis evaluated the role of the analgesic efficacy of the intraoperative use of the drug in the postoperative period. Patients scheduled to undergo elective spine surgery under general anaesthesia were screened for participation in the study. Patients of either gender in the age group of 30-60 years with expected duration of surgery of 1.5-2.5 hours and falling under American Society of Anaesthesiology (ASA) physical status I or II, were included. The exclusion criteria were 1. Patients having any severe systemic illness. 2. Long term use of certain medications. 3. Psychiatric illness. 4. Alcohol/drug abuse. 5. Patients on chronic opioid analgesic, tri cyclic anti-depressant (TCA), clonidine, mono-amine oxidase inhibitor (MAOI) therapy. 6. Heavy smoking habit. 7. Pregnancy and breast-feeding mothers. 8. History of allergic reactions to any drug. 9. Abnormal preoperative electrolyte concentrations. 10. Participation in any other clinical trial within 3 month. Eligible patients providing written voluntary informed consent $(n=60)$ were randomised into two equal groups: - group D (patients received dexmedetomidine $1 \mu \mathrm{g} / \mathrm{kg}$ in 10 mins, followed by $0.5 \mu \mathrm{g} / \mathrm{kg} / \mathrm{hr}$ ) and group C (control group- patients received equal volume of $0.9 \%$ normal saline in the same manner). Randomisation was done using computer generated random number table using block randomisation in blocks of 6 . The sample size estimation for the randomised controlled trial was calculated with the primary outcome of the primary analysis. The same sample size ( $n=60,30$ in each arm) was used for the secondary analysis and the power of the secondary analysis was estimated post-hoc. Anaesthesia was practised as per the prevailing standard of care. Induction of anaesthesia, maintenance of anaesthesia, analgesia and neuromuscular blockade was achieved with propofol, nitrous oxide and propofol, fentanyl and rocuronium respectively in the recommended doses. The details of randomisation method, sample size calculation, procedure of anaesthesia and the primary analysis is described in our previously published study. ${ }^{3}$ The study was approved by Institutional Ethics Committee, Calcutta National Medical College \& Hospital, Kolkata (No CNMC/ETHI/5042/P dated $16^{\text {th }}$ Dec 2010) and conducted from July 2011 to June 2012. Statistical analysis was done in SPSS version 23.0.

\section{Outcome measures}

- Visual Analogue Scale (VAS) score for pain at multiple time points in the first 24 hours of the post-operative period.

- Time to first rescue analgesic
- Back-up analgesic in the form of intramuscular diclofenac sodium

- Total requirement of analgesic $[\operatorname{tramadol}(\mathrm{mg} / \mathrm{kg})]$ in 24 hrs after operation

\section{Assessment of pain}

Patients were assessed for pain after shifting to post anaesthetic care unit from operation theatre by a resident not involved in the study using Visual Analog Scale (VAS) for pain at multiple time points. In VAS, pain was scored from $0-10(0=$ no pain, and $10=$ worse imaginable pain $)$ in a 10 point linear VAS. ${ }^{4}$ Pain was scored at 30 mins, $1 \mathrm{hr}$, 2 hrs, 6 hrs, 12 hrs and 24 hrs after operation.

\section{Assessment of analgesic efficacy \\ VAS score at multiple time points}

The VAS scores, which showed non-normal distribution, were compared between the two groups using nonparametric Mann-Whitney test at the observed time points in the two groups.

\section{Time to first rescue analgesia}

VAS score $\geq 5$ was treated with rescue analgesia, tramadol in a bolus of $1-2 \mathrm{mg} / \mathrm{kg}$ intravenous, repeated if necessary after $15 \mathrm{~min}$. Time to first rescue analgesic was defined as time from closure of skin to the first request or indication for supplemental analgesia because of VAS score greater than five. ${ }^{5}$ Kaplan-Meier survival curves were created for the two groups and comparison between the groups was achieved with log-rank test assuming equality of survivor functions. ${ }^{6}$

\section{Requirement of additional analgesic in the form of back-up analgesia}

If analgesia was still inadequate after 15 mins of second dose of tramadol, injection diclofenac sodium $75 \mathrm{mg}$ intramuscularly was administered as back up analgesic. The number of patients requiring back-up analgesia was compared between the two groups. For inferential statistics, Fischer's exact test was used to deduce measure of statistically significant difference between the two groups.

\section{Total dose of analgesic required during the first 24 hours after operation}

The total dose of administered tramadol during the first $24 \mathrm{hr}$ period following extubation was recorded. The cumulative dose of administered tramadol is inversely related with the analgesic efficacy of the administered agents. Data was expressed as mean \pm Standard deviation and statistical significance of intergroup difference was derived using independent sample t-test. 


\section{RESULTS}

\section{Baseline characteristics}

The demographic and clinical characteristics of the patients in the two arms are shown in Table 1. There was no statistically significant difference between the two arms with respect to the baseline characteristics.

\section{Analgesic efficacy}

The mean VAS scores (on a scale of 0 to 10) in the immediate post-operative period (30 $\mathrm{min})$ and after 1 , 2 and $6 \mathrm{hr}$ in the postoperative period for group D and group $C$ was 1.54, 2.33, 3.4, 3.67 and 5.87, 5.4, 4.64, 4.74 respectively. The $\mathrm{P}$ value for the difference in mean score between the two groups obtained using the non-parametric Mann-Whitney test was $<0.0001$. The VAS scores at 12 and $24 \mathrm{~h}$ were comparable in the two groups (4.44, 4.67 and 4.7, 4.64 for group D and group $C$ respectively), but at the expense of higher analgesic consumption in group C (Figure 1, Table 2).

\section{Time to first rescue analgesic}

The Kaplan-Meier survival curves (Figure 2) for the two groups show that the time to request for first rescue analgesic after surgery was longer in group D (Median-155, Range $=125-200)$ than group C (Median-25,

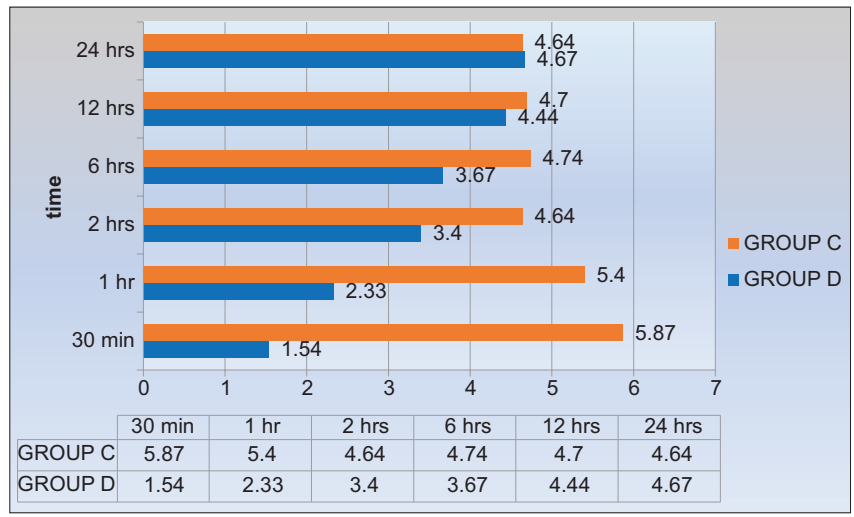

Figure 1: Comparison of VAS score between the two study groups at multiple time points in the post-operative period. Scores are expressed as means
Range $=10-45)$, the difference being statistically significant (P-value $<0.0001$ by log-rank test for equality in survivor function) (Table 3)

\section{Back-up analgesic}

The requirement of back-up analgesia during the first 24 hours was significantly lower in group D as compared with group $\mathrm{C}(\mathrm{P}$ value $=0.0008)$ (Table 3$)$.

\section{Total requirement of analgesic (tramadol)}

The total dose of tramadol as rescue analgesic during the first $24 \mathrm{~h}$ was significantly lower in group D (Mean $\pm \mathrm{SD}=6.14$, $0.55)$ as compared with group $C($ Mean $\pm S D=9.52,0.88)$. The $\mathrm{P}$ value was $<0.0001$ between the groups (Table 3 ).

\section{Power of the study}

The post-hoc power analysis for statistically significant difference between the groups at 5\% significance level for the different outcome parameters is shown in Table 3. The sample size of 60 (30 in each arm) which was calculated for the primary analysis (with power of $90 \%$ ) was also found to be adequately powered for the secondary analysis.

\section{DISCUSSION}

The primary aim of our study was to assess the postoperative analgesic efficacy of intraoperative use of dexmedetomidine in elective lumbar spine surgery under general anaesthesia. The results of our study show that the intra-operative use of the drug mitigates pain in the postoperative period and also reduce the requirement of the analgesic agents. The analgesic action of dexmedetomidine is not new in anaesthesia and the scientific evidence in favour of the drug as an supplementary analgesic is becoming stronger. ${ }^{7-13}$ In this article, we have evaluated the analgesic efficacy of the drug using multiple outcome parameters and compared the results with existing literature.

The time to first rescue analgesic was longer in group D $(\mathrm{P}<0.0001)$. Intraoperative infusion of dexmedetomidine reduced mean requirement of tramadol (rescue analgesic) in the first $24 \mathrm{hr}$. No patient in group D required

\begin{tabular}{|c|c|c|c|c|}
\hline SI. No. & Variable & Group D & Group C & $P$ value \\
\hline 01 & Age in years & $42.54 \pm 6.70$ & $41.94 \pm 3.75$ & 0.25 \\
\hline 02 & Body weight in Kgs & $57.4 \pm 6.80$ & $54.74 \pm 5.71$ & 0.25 \\
\hline 03 & Gender (Male/Female) & $21 / 9$ & $19 / 11$ & 0.78 \\
\hline 04 & Height in Cms & $158.97 \pm 5.57$ & $157.6 \pm 6.34$ & 0.39 \\
\hline 06 & Duration of surgery in minutes & $135 \pm 11.21$ & $129.5 \pm 14.16$ & 0.13 \\
\hline 07 & Type of Surgery (MD/PI) & $16 / 14$ & $18 / 12$ & 0.79 \\
\hline
\end{tabular}

ASA-PS- American Society of Anaesthesiology- Physical Status, MD- Microdiscectomy, PI-Pelvic Instrumentation 
injection diclofenac (back up analgesic). Patients of dexmedetomidine receiving group had less VAS score at $30 \mathrm{~min}, 1 \mathrm{hr}, 2 \mathrm{hr}$ and $6 \mathrm{hr}$ in the post-operative period. It has been reported that dexmedetomidine potentiates analgesia caused by fentanyl and reduces its dose requirements in humans during surgery. ${ }^{14}$ Turgut $\mathrm{N}$ et al stated that opioid requirements in the intraoperative period and in the postoperative period were reduced by dexmedetomidine..$^{15}$ Activation of the receptors in the brain and spinal cord inhibits neuronal firing causing sedation and analgesia. ${ }^{16-18}$ In general, presynaptic activation of $\alpha 2$ adrenoceptor inhibits the release of norepinephrine, terminating the propagation of pain signals. ${ }^{19,20}$ Nakagawa et al suggested that $\alpha 2$

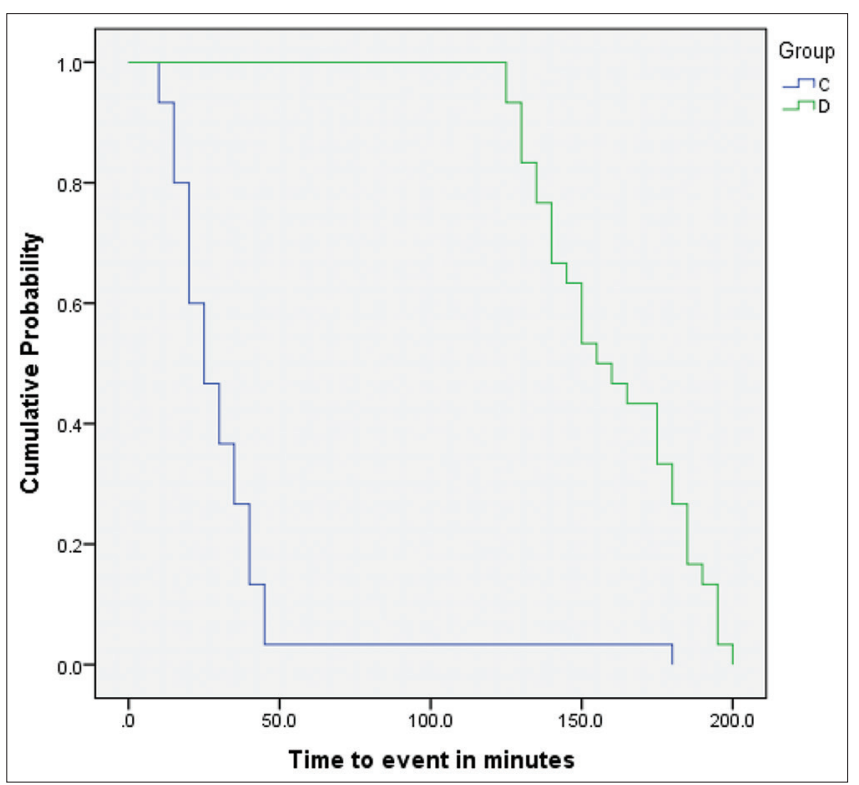

Figure 2: Kaplan-Meier curves showing the cumulative probability of non-requirement of rescue analgesic on the $y$-axis and the postoperative time on the $x$-axis

\begin{tabular}{|c|c|c|c|}
\hline PERIOD & GROUP D & GROUP C & $P$ value \\
\hline After 30 minutes & $1.54(0.57)$ & $5.87(0.97)$ & $<0.0001$ \\
\hline After 1 hour & $2.33(0.54)$ & $5.4(0.72)$ & $<0.0001$ \\
\hline After 2 hours & $3.4(0.62)$ & $4.64(0.76)$ & $<0.0001$ \\
\hline After 6 hours & $3.67(0.88)$ & $4.74(0.83)$ & $<0.0001$ \\
\hline After 12 hours & $4.44(0.77)$ & $4.7(0.6)$ & 0.19 \\
\hline After 24 hours & $4.67(0.92)$ & $4.64(0.61)$ & 0.83 \\
\hline
\end{tabular}

adrenergic mechanisms are involved in the modulation of nociception, at the level of spinal noradrenergic systems. ${ }^{21}$ The means to assess postoperative pain control was the time to first analgesic consumption, the total amount of analgesic consumed in the first $24 \mathrm{hr}$ period after surgery and the VAS scores at different times in the first postoperative day. Time to request for rescue analgesic for the first time was considered as the duration of postoperative analgesia. The time to first rescue analgesic in the post-operative period was significantly increased by dexmedetomidine in our study with a $\mathrm{P}$ value $<0.0001$. This longer duration of postoperative analgesia was achieved by intraoperative dexmedetomidine infusion as its half-life is 1.8-2 hrs. Mean requirement of tramadol (rescue analgesic) in the first $24 \mathrm{hr}$ was also lesser in dexmedetomidine receiving group. VAS score remained lower in dexmedetomidine treated group in immediate postoperative period at $30 \mathrm{~min}, 1$ hour, 2 hours and 6 hours interval ( $p$ value $<0.0001$ ). No patient in group $\mathrm{D}$ required injection diclofenac (back-up analgesic) in contrast to ten (10) patients in group C. In the control group, the delayed onset and prolonged duration of action of tramadol and diclofenac resulted in better postoperative analgesia subsequently in the later part of the postoperative period. This was reflected in the comparative values of the VAS scores at 12 and $24 \mathrm{~h}$ postoperatively, but at the expense of higher analgesic consumption in group C. This result corroborates with the finding of previous studies. Feld et al.148 in 2006, compared dexmedetomidine to fentanyl and reported that dexmedetomidine provided both stable perioperative haemodynamics and postoperative analgesia, thus reducing the use of morphine in the postoperative period. ${ }^{22}$ In another study Gurbet A et al in 2006 reported that continuous i.v. dexmedetomidine during abdominal surgery provided effective postoperative analgesia and reduced postoperative morphine requirements without increasing the incidence of side effects. ${ }^{13}$ Naik et al, in a study involving deformity correction spine surgery in adult patients, also found that there was significant reduction of analgesic requirement in intra-operative analgesia in the dexmedetomidine arm compared to placebo. ${ }^{23}$ No significant difference in analgesic requirement was observed between the dexmedetomidine and placebo arm in the post-operative period. The intraoperative and post-operative analgesia was provided with fentanyl and hydromorphone respectively. ${ }^{23}$ Conversely, Garg et al,

\section{Table 3: Comparison of analgesic efficacy between the two groups using multiple outcome parameters}

\begin{tabular}{|c|c|c|c|c|c|c|}
\hline & Efficacy parameter for analgesic action & Group D & Group C & Statistical test & $P$ value & Post-hoc power \\
\hline 01 & Time (minutes) to first rescue analgesic ( & $155,125-200$ & $25,10-45$ & Log & $<0$. & 98.31 \\
\hline 02 & Total dose of tramadol $(\mathrm{mg} / \mathrm{kg})$ in $24 \mathrm{hrs}$ (Mean, SD) & $6.14,0.55$ & $9.52,0.88$ & Independent t test & $<0.0001$ & 100 \\
\hline 03 & Proportion of patients requiring back-up analgesic & $0 / 30$ & $10 / 30$ & Fischer's exact test & $=0.0008$ & 96.98 \\
\hline
\end{tabular}


found dexmedetomidine equally efficacious to ketamine for post-operative analgesia in spine surgery, both of which were superior to placebo in terms of requirement of rescue analgesia. ${ }^{24}$ The superior pain relief accorded by intravenous dexmedetomidine prompted researchers to explore the efficacy of the drug through alternate routes like local infiltration and intrathecal. Daiki et al, found that the combined infiltration of dexmedetomidine and ropivacaine at the local site in lumbar discectomy surgery, provided better pain relief on the Visual Analog Scale compared to ropivacaine alone. ${ }^{25}$ Intrathecal administration along with local anaesthetics has been found to prolong duration of anaesthesia and reduce the requirement of analgesia in various type of surgeries. ${ }^{26-28}$

\section{CONCLUSION}

Dexmedetomidine provides effective postoperative analgesia and reduces tramadol requirements in elective spine surgery performed under general anaesthesia.

\section{ACKNOWLEDGEMENT}

The authors sincerely thank the Department of Anaesthesiology and Neuro-surgery for support and cooperation all throughout the conduct of the study.

\section{REFERENCES}

1. Keating GM, Hoy SM and Lyseng-Williamson KA. Dexmedetomidine: a guide to its use for sedation in the US. Clin Drug Investig. 2012; 32(8):561-567.

https://doi.org/10.1007/BF03261910

2. Davy A, Fessler J, Fischler M and LE Guen M. Dexmedetomidine and general anesthesia: a narrative literature review of its major indications for use in adults undergoing non-cardiac surgery. Minerva Anestesiol. 2017; 83(12):1294-1308.

3. Ray M, Dalai CK, Ahmed SN and Acharjee A. A triple blind, randomised, placebo controlled, add-on trial to assess the efficacy and safety of dexmedetomidine in facilitation of general anaesthesia in patients undergoing elective spine surgery. Asian J Med Sci. 2021; 12 (3):

https://doi.org/10.3126/ajms.v12i3.32831

4. Heller GZ, Manuguerra M and Chow R. How to analyze the Visual Analogue Scale: Myths, truths and clinical relevance. Scand J Pain. 2016; 13:67-75.

https://doi.org/10.1016/j.sjpain.2016.06.012

5. Bielka K, Kuchyn I, Babych V, Martycshenko K and Inozemtsev O. Dexmedetomidine infusion as an analgesic adjuvant during laparoscopic cholecystectomy: a randomized controlled study. BMC Anesthesiol. 2018; 18(1):44.

https://doi.org/10.1186/s12871-018-0508-6

6. Capici F, Ingelmo PM, Davidson A, Sacchi CA, Milan B, Rota Sperti $L$, et al. Randomized controlled trial of duration of analgesia following intravenous or rectal acetaminophen after adenotonsillectomy in children. $\mathrm{Br} \mathrm{J}$ Anaesth. 2008; 100(2): 251-255. https://doi.org/10.1093/bja/aem377

7. Tanskanen PE, Kyttä JV, Randell TT and Aantaa RE. Dexmedetomidine as an anaesthetic adjuvant in patients undergoing intracranial tumour surgery: a double-blind, randomized and placebo-controlled study. Br J Anaesth. 2006; 97(5):658-665.

https://doi.org/10.1093/bja/ael220

8. Keniya VM, Ladi $S$ and Naphade R. Dexmedetomidine attenuates sympathoadrenal response to tracheal intubation and reduces perioperative anaesthetic requirement. Indian $\mathrm{J}$ Anaesth. 2011; 55(4):352-357.

https://doi.org/10.4103/0019-5049.84846

9. Tang C and Xia Z. Dexmedetomidine in perioperative acute pain management: a non-opioid adjuvant analgesic. J Pain Res. 2017; 10:1899-1904.

https://doi.org/10.2147/JPR.S139387

10. Lee YYS, Wong SM and Hung CT. Dexmedetomidine infusion as a supplement to isoflurane anaesthesia for vitreoretinal surgery. Br J Anaesth. 2007; 98(4):477-483.

https://doi.org/10.1093/bja/aem040

11. Aho $M$, Erkola $O$, Kallio $A$, Scheinin $H$ and Korttila $K$. Dexmedetomidine infusion for maintenance of anesthesia in patients undergoing abdominal hysterectomy. Anesth Analg. 1992; 75(6):940-946. https://doi.org/10.1213/00000539-199212000-00012

12. Aantaa $R$, Kanto $J$, Scheinin $M$, Kallio $A$ and Scheinin $H$. Dexmedetomidine, an alpha 2-adrenoceptor agonist, reduces anesthetic requirements for patients undergoing minor gynecologic surgery. Anesthesiology. 1990; 73(2):230-235. https://doi.org/10.1097/00000542-199008000-00007

13. Gurbet A, Basagan-Mogol E, Turker G, Ugun F, Kaya FN and Ozcan B. Intraoperative infusion of dexmedetomidine reduces perioperative analgesic requirements. Can J Anaesth J Can Anesth. 2006; 53(7):646-652. https://doi.org/10.1007/BF03021622

14. Scheinin $B$, Lindgren $L$, Randell $T$, Scheinin $H$ and Scheinin $M$. Dexmedetomidine attenuates sympathoadrenal responses to tracheal intubation and reduces the need for thiopentone and peroperative fentanyl. $\mathrm{Br} \mathrm{J}$ Anaesth. 1992; 68(2):126-131. https://doi.org/10.1093/bja/68.2.126

15. Turgut N, Turkmen A, Gökkaya S, Altan A and Hatiboglu MA. Dexmedetomidine-based versus fentanyl-based total intravenous anesthesia for lumbar laminectomy. Minerva Anestesiol. 2008; 74(9):469-474.

16. Coursin DB, Coursin DB and Maccioli GA. Dexmedetomidine. Curr Opin Crit Care. 2001; 7(4):221-226. https://doi.org/10.1097/00075198-200108000-00002

17. Sakaguchi Y, Takahashi S. [Dexmedetomidine]. Masui. 2006; 55(7):856-863.

18. Smith $\mathrm{H}$ and Elliott J. Alpha(2) receptors and agonists in pain management. Curr Opin Anaesthesiol. 2001; 14(5):513-518. https://doi.org/10.1097/00001503-200110000-00009

19. Li $\mathrm{X}$ and Eisenach JC. alpha2A-adrenoceptor stimulation reduces capsaicin-induced glutamate release from spinal cord synaptosomes. J Pharmacol Exp Ther. 2001; 299(3):939-944.

20. Fürst $S$. Transmitters involved in antinociception in the spinal cord. Brain Res Bull. 1999;48(2):129-141. https://doi.org/10.1016/S0361-9230(98)00159-2

21. Nakagawa I, Omote K, Kitahata LM, Collins JG and Murata K. Serotonergic mediation of spinal analgesia and its interaction with noradrenergic systems. Anesthesiology. 1990; 73(3): 474-478. 
https://doi.org/10.1097/00000542-199009000-00017

22. Feld JM, Hoffman WE, Stechert MM, Hoffman IW and Ananda RC. Fentanyl or dexmedetomidine combined with desflurane for bariatric surgery. J Clin Anesth. 2006; 18(1):24-28. https://doi.org/10.1016/j.jclinane.2005.05.009

23. Naik BI, Nemergut EC, Kazemi A, Fernández L, Cederholm SK, McMurry TL, et al. The Effect of Dexmedetomidine on Postoperative Opioid Consumption and Pain After Major Spine Surgery. Anesth Analg. 2016; 122(5):1646-1653. https://doi.org/10.1213/ANE.0000000000001226

24. Garg $S$ and Singh R. Need for integration of gender equity in family planning services. Indian J Med Res. 2014; 140(Suppl 1):S147-S151.

25. Daiki M, Najar M, Chkili R, Rafrafi A, Gabsia AB, Labbene I, et al. Postoperative analgesia after wound infiltration with Dexmedetomidine and Ropivacaine versus Ropivacaine alone for lumbar discectomies: a randomized-controlled trial. Tunis Med. 2019; 97(12):1375-1382.

26. Salem RA, Darweesh EI, Wanis MAand Mohamed AA. Evaluation of the effects of intrathecal bupivacaine-dexmedetomidine for lumbar spine fusion: a double blinded randomized controlled study. Eur Rev Med Pharmacol Sci. 2015; 19(23):4542-4548.

27. Gautam B, Tabdar S and Shrestha U. Comparison of Fentanyl and Dexmedetomidine as Intrathecal Adjuvants to Spinal Anaesthesia for Abdominal Hysterectomy. J Nepal Med Assoc. 2018; 56(213):848-855.

https://doi.org/10.31729/jnma.3739

28. Gautam B, Lama SM and Sharma M. Effects of Adding Intrathecal Dexmedetomidine to Hyperbaric Bupivacaine for Saddle Spinal Block in Adults Undergoing Peri-anal Surgeries. J Nepal Health Res Counc. 2018; 16(1):43-48.

https://doi.org/10.3126/jnhrc.v16i1.19362

Author's contribution:

MR, CKD, AA - Concept and design of the study; prepared first draft of manuscript; MR, CKD, AA, SNA - Interpreted the results; reviewed the literature and manuscript preparation; SNA, CKD, MR - Statistically analysis and interpretation of results, preparation of manuscript and revision of the manuscript.

Work Attributed to:

Calcutta National Medical College \& Hospital, Kolkata, India.

Orcid ID:

Dr Madhumita Ray - (D) https://orcid.org/0000-0002-2882-5405

Dr Chanchal Kumar Dalai - id https://orcid.org/0000-0002-5406-3440

Dr Shah Newaz Ahmed - (D) https://orcid org/0000-0003-0083-346X

Source of Funding: None, Conflict of Interest: None. 